\title{
Parabolic fixed points, invariant curves and action-angle variables
}

\author{
DOV AHARONOV AND URI ELIAS \\ Department of Mathematics, Technion, Israel Institute of Technology, \\ Haifa 32000, Israel
}

(Received 26 July 1988)

\begin{abstract}
A fixed point of an area-preserving mapping of the plane is called elliptic if the eigenvalues of its linearization are of unit modulus but not \pm 1 ; it is parabolic if both eigenvalues are 1 or -1 . The elliptic case is well understood by Moser's theory. Here we study when is a parabolic fixed point surrounded by closed invariant curves. We approximate our mapping $T$ by the phase flow of an Hamiltonian system. A pair of variables, closely related to the action-angle variables, is used to reduce $T$ into a twist mapping. The conditions for $T$ to have closed invariant curves are stated in terms of the Hamiltonian.
\end{abstract}

\section{Introduction}

Consider an area-preserving mapping of the plane which has a fixed point and let $\lambda_{1}, \lambda_{2}$ be the eigenvalues of its linearization at this point. $\lambda_{1}, \lambda_{2}$ are either both real or are complex conjugates and $\lambda_{1} \lambda_{2}=1$. If $\lambda_{1}, \lambda_{2}$ are real and not \pm 1 , the fixed point is called hyperbolic and it cannot be stable. If $\lambda_{1}=\bar{\lambda}_{2},\left|\lambda_{1}\right|=\left|\lambda_{2}\right|=1$ and $\lambda_{1}$, $\lambda_{2}$ are not \pm 1 , the fixed point is called elliptic. This case is well understood by Moser's theory and it is known that if the $\lambda$ 's are not $k$ th root of the unity plus some mild condition, the fixed point is stable and it is surrounded by closed invariant curves $[2,3]$. Finally, if $\lambda_{1}=\lambda_{2}=1$, the fixed point is called parabolic. This case will be studied here.

So we consider an area-preserving mapping

$$
\begin{aligned}
& x_{1}=x+P(x, y), \\
& y_{1}=y+Q(x, y),
\end{aligned}
$$

where $P(x, y), Q(x, y)$ are real-analytic functions,

$$
P(x, y), Q(x, y)=O\left(r^{h}\right), \quad r^{2}=x^{2}+y^{2},
$$

$h>1$, near $(0,0)$ and

$$
P^{2}+Q^{2} \neq 0
$$

in a small, punctured neighbourhood of $(0,0)$. The origin is an isolated parabolic fixed point. Our aim is to obtain conditions which guarantee that this fixed point is surrounded by closed invariant curves. 
Our method is based on the reduction of the mapping (1.1) to a twist mapping. We shall suggest the system of Hamiltonian differential equations

$$
d x / d t=-H_{y}, \quad d y / d t=H_{x}
$$

with

$$
H(x, y) \stackrel{\text { def }}{\equiv} \int_{(0,0)}^{(x, y)}\left[Q-\frac{1}{2}\left(P Q_{x}-P_{x} Q\right)\right] d x-\left[P+\frac{1}{2}\left(P Q_{y}-P_{y} Q\right)\right] d y
$$

whose phase flow $g^{1}:(x(0), y(0)) \rightarrow(x(1), y(1))$ approximates the mapping (1.1) very well. This approximation is used to introduce a new pair of variables, $\rho, \theta$, which reduce (1.1) into a twist mapping.

After the completion of our manuscript we became aware of the works $[4,5]$ of C. Simo. Let $g\left(x, y_{1}\right)$ be the generating function of $(1.1)$, which is defined by

$$
x_{1}=\partial g\left(x, y_{1}\right) / \partial y, \quad y=\partial g\left(x, y_{1}\right) / \partial x
$$

and put $g\left(x, y_{1}\right)=x y_{1}+G\left(x, y_{1}\right)$. Simo claims that the parabolic fixed point of (1.1) is surrounded by closed invariant curves if and only if $G\left(x, y_{1}\right)$ has a strict extremum there. This had been done by the same idea utilized here, namely a reduction of the given mapping into a twist mapping by a time unit flow $g^{1}$ of some Hamiltonian system. Thus, our method is not new. We choose, naturally, the angle variable $\theta$ as in [5] and Proposition 2, for example, is identical with Simo's argument. However, the novelty of this work is the choice of the Hamiltonian. While Simo chooses the Hamiltonian to be $G\left(x, y_{1}\right)$, whose existence is guaranteed by the implicit function theory, our $H(x, y)$ is defined explicitly and the assumptions can be practically verified. In the most simple case, when a first approximation of the generating function suffices to apply Simo's theorem, the result is the same as our simplest particular case (Theorem 4.1). In more complicated cases, our result is more applicable but gives only sufficient conditions for stability. Note that some details in Simo's argument should be carefully reviewed.

Before we begin our work, we present two graphic examples which demonstrate the difficulties and the beauty of the subject. The examples will be discussed rigorously in $\$ 4$.

Example 1. The area-preserving mapping

$$
\begin{aligned}
& x_{1}=x+y^{2 n-1}, \\
& y_{1}=y-\left(x+y^{2 n-1}\right)^{2 n-1},
\end{aligned}
$$

is of type (1.1). The results of some numerical experiments for $2 n-1=3$ are shown in figure 1. Each curve is generated by several hundred iterations of an initial point.

Example 2. The mapping

$$
T: \begin{aligned}
& x_{1}=-x+y^{2} \\
& y_{1}=-y+x_{1}^{2}
\end{aligned}
$$

is area-preserving and its second iteration $S=T^{2}$

$$
\begin{aligned}
& x_{1}=x-2 x^{2} y+4 x y^{3}+x^{4}-2 y^{5}-4 x^{3} y^{2}+\cdots+y^{8}, \\
& y_{1}=y+2 x y^{2}-4 x^{3} y-y^{4}+2 x^{5}+8 x^{2} y^{3}+\cdots+y^{16},
\end{aligned}
$$

is of the form (1.1). Numerical experiments hint that the parabolic fixed point $(0,0)$ of $S$ is surrounded by closed invariant curves. See figure 2 . 


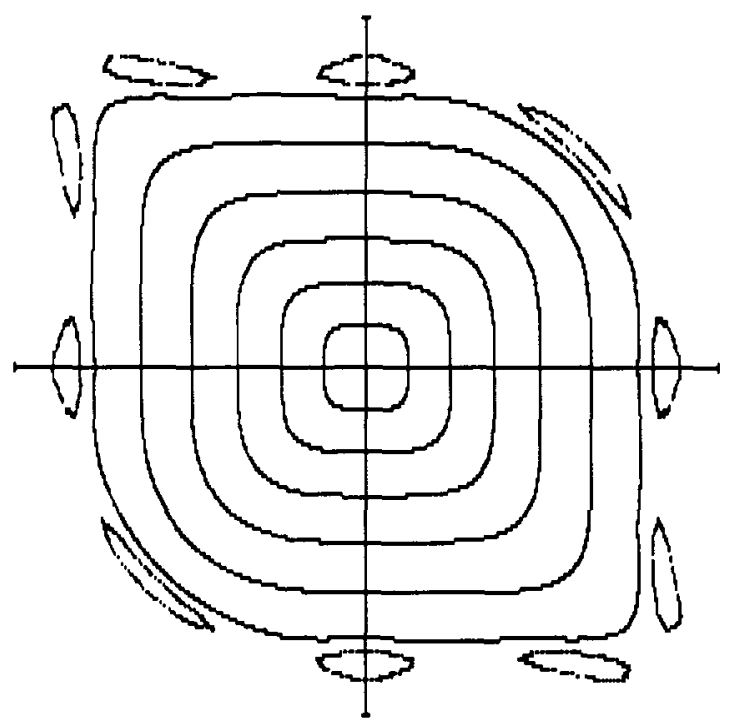

FIGURE 1. Invariant curves of Example 1.

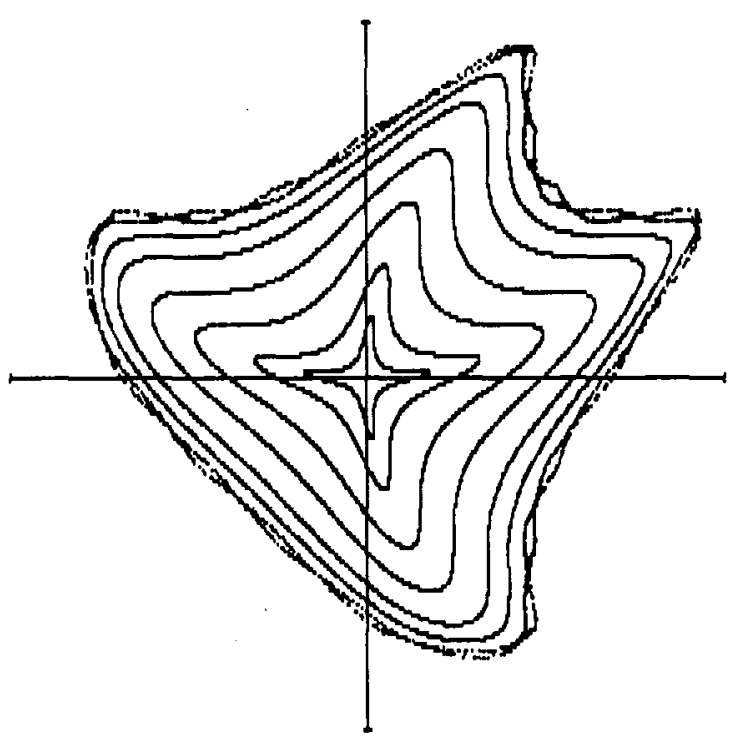

FIGURE 2. Invariant curves of Example 2.

\section{An Hamiltonian and new variables}

The main tool to establish the existence of invariant curves is Moser's theorem about twist mappings [2, Theorem 3; 3, \& 32]:

Given the mapping

$$
\begin{aligned}
& \theta_{1}=\theta+\gamma \rho+f(\rho, \theta), \\
& \rho_{1}=\rho+g(\rho, \theta) .
\end{aligned}
$$


in the annulus $a<\rho<b,-\infty<\theta<\infty, b-a \geq 1$, where $f, g$ are real analytic and periodic in $\theta$ and every closed curve sufficiently close to $\rho=$ constant intersects its image curve. For each $\varepsilon>0$ there exists $\delta$, independent of $\gamma$, such that if

$$
|f|+|g|<\gamma \delta
$$

then the mapping admits infinitely many invariant curves of the form

$$
\rho=v(t), \quad \theta=t+u(t),
$$

with $u, v$ real analytic, periodic functions and $|u(t)|,|v(t)-\omega|<\varepsilon$.

Our method is based on the reduction of the mapping (1.1) to a twist mapping. We shall suggest a system of Hamilton differential equations whose phase flow approximates the mapping (1.1). This continuous approximation is used to introduce a new pair of variables, $\theta, \rho$, which will satisfy all the conditions of Moser's theory.

Proposition 2.1. The mapping (1.1) is area peserving if and only if the system

$$
\begin{aligned}
& x^{\prime}=P+\frac{1}{2}\left(P Q_{y}-P_{y} Q\right), \\
& y^{\prime}=Q-\frac{1}{2}\left(P Q_{x}-P_{x} Q\right),
\end{aligned}
$$

generates an area-preserving mapping $g^{t}:(x(0), y(0)) \rightarrow(x(t), y(t))$.

Proof. The mapping (1.1) is area preserving if

$$
J=\frac{\partial\left(x_{1}, y_{1}\right)}{\partial(x, y)}=\left|\begin{array}{cc}
1+P_{x} & P_{y} \\
Q_{x} & 1+Q_{y}
\end{array}\right|=1+P_{x}+Q_{y}+P_{x} Q_{y}-P_{y} Q_{x} \equiv 1
$$

That is,

$$
P_{x}+Q_{y}+P_{x} Q_{y}-P_{y} Q_{x}=0
$$

or

$$
\left(P+\frac{1}{2}\left(P Q_{y}-P_{y} Q\right)\right)_{x}+\left(Q-\frac{1}{2}\left(P Q_{x}-P_{x} Q\right)\right)_{y}=0,
$$

which guarantees that the system (2.2) generates an area-preserving mapping $g^{\prime}$ and vice versa.

Consequently we define

$$
H(x, y) \stackrel{\text { def }}{=} \int_{(0,0)}^{(x, y)}\left[Q-\frac{1}{2}\left(P Q_{x}-P_{x} Q\right)\right] d x-\left[P+\frac{1}{2}\left(P Q_{y}-P_{y} Q\right)\right] d y,
$$

where, by (2.3), the integral is independent of the path of the integration. By this notation, (2.2) can be written as system of Hamilton canonical equations

$$
\begin{aligned}
& d x / d t=-H_{y}, \\
& d y / d t=H_{x}
\end{aligned}
$$

and its trajectories are $H(x, y)=$ constant.

From now on, we shall assume that $H(x, y)=\lambda$ are closed curves which surround the origin for sufficiently small values of $\lambda$. This implies, among other things, that the corresponding solutions of (2.4) are periodic. 
We may get an idea about the form of $H$ if the area-preserving mapping (1.1) is written in a neighborhood of $(0,0)$ as

$$
\begin{aligned}
& x_{1}=x+p(x, y)+\hat{p}(x, y), \\
& y_{1}=y+q(x, y)+\hat{q}(x, y),
\end{aligned}
$$

where $p(x, y), q(x, y)$ are polynomials homogeneous of degree $h, h>1$, and $\hat{p}, \hat{q}$ are such that $\hat{p}(x, y), \hat{q}(x, y)=O\left(r^{h+1}\right)$ near $r=0$. By considering the lowest order terms of (2.3), we get that

$$
p_{x}+q_{y}=0 \text {. }
$$

This, together with the homogeneity of $p, q$ of order $h$, implies that

$$
(x q-y p)_{x}=x q_{x}+q-y p_{x}=\left(x q_{x}+y q_{y}\right)+q=(h+1) q,
$$

and $(x q-y p)_{y}=-(h+1) p$. Thus $H_{x}=q+O\left(r^{h+1}\right), H_{y}=-p+O\left(r^{h+1}\right)$ imply

$$
H(x, y)=(x q-y p) /(h+1)+O\left(r^{h+2}\right) \text {. }
$$

First we estimate how well does the phase flow of (2.4) approximates the mapping (1.1).

Proposition 2. Consider the mapping (1.1) which maps $(x, y)$ to $\left(x_{1}, y_{1}\right)$ and the mapping $g^{\prime}:(x, y) \rightarrow(x(t), y(t))$ which is generated by (2.4). If $P, Q=O\left(r^{h}\right)$, then

$$
x(1)-x_{1}, y(1)-y_{1}=O\left(r^{3 h-2}\right) .
$$

Proof. Equations (2.4) are real-analytic and according to our assumptions all their solutions are periodic. Hence $x(t), y(t)$ are real-analytic for all $t$,

$$
x(t)=x(0)+x^{\prime}(0) t+x^{\prime \prime}(0) t^{2} / 2+\cdots .
$$

Now

$$
\begin{aligned}
& x^{\prime}=P+\frac{1}{2}\left(P Q_{y}-P_{y} Q\right), \\
& x^{\prime \prime}=\left[P+\frac{1}{2}\left(P Q_{y}-P_{y} Q\right)\right]_{x} x^{\prime}+\left[Q-\frac{1}{2}\left(P Q_{x}-P_{x} Q\right)\right]_{y} y^{\prime}
\end{aligned}
$$

By (2.3)

$$
\begin{aligned}
{\left[P+\frac{1}{2}\left(P Q_{y}-P_{y} Q\right)\right]_{x} } & =P_{x}+\frac{1}{2}\left(P_{x} Q_{y}-P_{y} Q_{x}\right)+\frac{1}{2}\left(P Q_{x y}-P_{x y} Q\right) \\
& =-Q_{y}-\frac{1}{2}\left(P_{x} Q_{y}-P_{y} Q_{x}\right)+\frac{1}{2}\left(P Q_{x y}-P_{x y} Q\right)
\end{aligned}
$$

so

$$
\begin{aligned}
x^{\prime \prime}= & {\left[-Q_{y}-\frac{1}{2}\left(P_{x} Q_{y}-P_{y} Q_{x}\right)+\frac{1}{2}\left(P Q_{x y}-P_{x y} Q\right)\right]\left[P+\frac{1}{2}\left(P Q_{y}-P_{y} Q\right)\right] } \\
& +\left[P_{y}+\frac{1}{2}\left(P Q_{y y}-P_{y y} Q\right)\right]\left[Q-\frac{1}{2}\left(P Q_{x}-P_{x} Q\right)\right] \\
= & -P Q_{y}+P_{y} Q+O\left(r^{3 h-2}\right)
\end{aligned}
$$

and similarly, $x^{(i)}(t)=O\left(r^{3 h-2}\right), i \geq 3$. Thus

$$
x(1)=x(0)+x^{\prime}(0)+x^{\prime \prime}(0) / 2+\cdots=x+P+O\left(r^{3 h-2}\right) .
$$

$y(1)$ is treated similarly.

The next proposition will show that $H(x, y)=\lambda$ is mapped by (1.1) quite close to itself. 
Proposition 3. $H(x+P, y+Q)-H(x, y)=O\left(r^{4 h-2}\right)$.

\section{Proof.}

$$
\begin{aligned}
H(x & +P, y+Q)-H(x, y) \\
& =\left[H_{x} P+H_{y} Q\right]+\frac{1}{2}\left[H_{x x} P^{2}+2 H_{x y} P Q+H_{y y} Q^{2}\right]+\frac{1}{6}\left[H_{x x x} P^{3}+\cdots\right]+\cdots
\end{aligned}
$$

Here

$$
\begin{gathered}
H_{x}=Q-\frac{1}{2}\left(P Q_{x}-P_{x} Q\right), \quad H_{y}=P+\frac{1}{2}\left(P Q_{y}-P_{y} Q\right) \\
H_{x x}=Q_{x}-\frac{1}{2}\left(P Q_{x x}-P_{x x} Q\right), \quad H_{y y}=-P_{y}-\frac{1}{2}\left(P Q_{y y}-P_{y y} Q\right) \\
2 H_{x y}=\left(H_{x}\right)_{y}+\left(H_{y}\right)_{x}=Q_{y}-P_{x}+P_{x y} Q-P Q_{x y} \\
H_{x x x}=Q_{x x}-\frac{1}{2}\left(P_{x} Q_{x x}+\cdots\right), \quad \text { etc. }
\end{gathered}
$$

So

$$
\begin{aligned}
\Delta H= & {\left[Q-\frac{1}{2}\left(P Q_{x}-P_{x} Q\right)\right] P+\left[P+\frac{1}{2}\left(P Q_{y}-P_{y} Q\right)\right] Q+\frac{1}{2}\left[Q_{x}-\frac{1}{2}\left(P Q_{x x}-P_{x x} Q\right)\right] P^{2} } \\
& +\frac{1}{2}\left[Q_{y}-P_{x}+P_{x y} Q-P Q_{x y}\right] P Q+\frac{1}{2}\left[-P_{y}-\frac{1}{2}\left(P Q_{y y}-P_{y y} Q\right)\right] Q^{2} \\
& +\frac{1}{6}\left[Q_{x x} P^{3}+3 Q_{x y} P^{2} Q-3 P_{x y} P Q^{2}-P_{y y} Q^{3}\right]+O\left(r^{5 h-3}\right) \\
= & -\frac{1}{12}\left[Q_{x x} P^{3}-3 P_{x x} P^{2} Q+3 Q_{y y} P Q^{2}-P_{y y} Q^{3}\right]+O\left(r^{5 h-3}\right)=O\left(r^{4 h-2}\right) .
\end{aligned}
$$

Now we begin a series of heuristic arguments which lead us to the choice of new variables $\rho, \theta$. Since in the theory of Moser, the invariant curves of the twist mapping are located near the curves $\rho=$ constant, and since we expect that the trajectories $H(x, y)=$ constant are close to the invariant curves, we shall seek a $\rho(x, y)$ which is constant on $H=$ constant. Thus we choose

$$
\rho=\psi(H(x, y)),
$$

where $\psi$ will be determined later.

The next heuristic considerations will lead us to the choice of $\theta(x, y)$. Since in the twist mapping $\theta_{1}-\theta \cong \rho$, we try to choose $\theta(x, y)$ so that

$$
\theta(x+P, y+Q)-\theta(x, y) \cong \theta_{x} P+\theta_{y} Q \cong \rho .
$$

But as $H_{x} \cong Q,-H_{y} \cong P$, we want $\theta(x, y)$ to satisfy $-\theta_{x} H_{y}+\theta_{y} H_{x}=\rho=\psi(H)$. Let us assume from now on that the closed curves $H=$ constant are starlike and define $\theta(x, y)$ as the solution of the Cauchy problem

$$
\begin{gathered}
-\theta_{x} H_{y}+\theta_{y} H_{x}=\psi(H), \\
\theta(x, 0)=0, \quad x>0 .
\end{gathered}
$$

The integral surface $\theta=\theta(x, y)$ of (2.9) consist of characteristic curves which satisfy

$$
d x /\left(-H_{y}\right)=d y / H_{x}=d \theta / \psi(H)
$$

and pass through the $x$-axis. The projections of the characteristic curves on the $x-y$ plane are the solutions of

$$
\begin{gathered}
d x / d \theta=-H_{y} / \psi(H), \\
d y / d \theta=H_{x} / \psi(H), \\
x(0)=x_{0}, y(0)=0, \quad x_{0}>0
\end{gathered}
$$

and the trajectories of this system are, of course, $H(x, y)=$ constant. Consequently, 
$\theta(x, y)$ is the time required for the solution $(x(\theta), y(\theta))$ of $(2.10)$ to arrive from the unique suitable point on the positive $x$-axis to the point $(x, y)$ while passing an arc of the curve $H(x, y)=$ constant. Since $H_{x}^{2}+H_{y}^{2}=\left(P^{2}+Q^{2}\right)(1+o(1)) \neq 0$ in a small punctured neighbourhood of the origin, $(2.10)$ has no critical points except $(0,0)$ and $(x(\theta), y(\theta))$ traverses the trajectory $H(x, y)=\lambda$ in one direction. Thus we obtain a multi-valued function $\theta(x, y)$ in every ring $\lambda_{1} \leq H \leq \lambda_{2}$.

On the other hand, (2.4) and (2.10) have the same trajectories $H(x, y)=$ constant and their solutions differ only by their different parametrizations $\theta$, $t$, which are related by

$$
d \theta / d t=\psi(H)=\rho .
$$

So on every trajectory $H=$ constant,

$$
\theta=\psi(H) t=\rho t .
$$

In order that the twist mapping will be periodic in $\theta$, we want the solution $\theta$ of (2.9) to increase by a fixed number, say 1 , when $(x, y)$ surrounds the origin once along $H=\lambda$. This is the case if we choose

$$
\psi(\lambda)=1 / T(\lambda)
$$

where $T(\lambda)$ is the period of the solution of (2.4) which traverses $H=\lambda$. Thus

$$
\rho(x, y)=1 / T(H(x, y)) \text {. }
$$

By (2.11) we have the representation

$$
\theta(x, y)=\rho(x, y) \int_{H=\lambda}^{(x, y)} \frac{-y d x+x d y}{x H_{x}+y H_{y}},
$$

where the integration is along the curve $H=\lambda$ from the positive $x$-axis to the point $(x, y)$. Indeed, if we parametrize the integral in (2.13) by the solutions $x=x(t)$, $y=y(t)$ of (2.4), it becomes $\int^{(x, y)} d t$, the time required for the solution $(x(t), y(t))$ of $(2.4)$ to arrive to the point $(x, y)$.

The area $A(\lambda)$ of the interior of the closed curve $H(x, y)=\lambda$ is closely related to our discussion. The differential of the arclength on $H=\lambda$ is $d s=\left[x^{\prime 2}+y^{\prime 2}\right]^{1 / 2} d t=$ $\left[H_{x}^{2}+H_{y}^{2}\right]^{1 / 2} d t$ and the length of the normal to $H=\lambda$, which connects $H=\lambda$ to $H=\lambda+\Delta \lambda$, is $\Delta \lambda /|\operatorname{grad} H|=\Delta \lambda /\left[H_{x}^{2}+H_{y}^{2}\right]^{1 / 2}$. Hence, the area of the ring $\lambda \leq H \leq$ $\lambda+\Delta \lambda$ is

$$
A(\lambda+\Delta \lambda)-A(\lambda) \cong \int_{0}^{T(\lambda)} n d s=\Delta \lambda T(\lambda)
$$

Thus

$$
d A / d \lambda=T(\lambda) .
$$

For a different proof, see $[1$, p. 282].

In our case we have

$$
T(\lambda) \rightarrow \infty \quad \text { as } \lambda \rightarrow 0 .
$$

For, if $P(x, y), Q(x, y)=O\left(r^{h}\right), h>1$, then $H(x, y)=O\left(r^{h+1}\right)$ near the origin. On a curve $H=\lambda$ we have $\lambda \leq c_{1} r^{h+1}$ and so $A(\lambda) \geq c_{2} \lambda^{2 /(h+1)}$. Since $A(\lambda)$ is analytic and $A(0)=0$, it follows that $T(\lambda)=A^{\prime}(\lambda) \rightarrow \infty$. 
If we put in (2.9) $\psi(H)=1 / T(H)=1 /\left.A^{\prime}(\lambda)\right|_{\lambda=H}$, it may be written as

$$
-\frac{\partial \theta}{\partial x} \frac{\partial A(H(x, y))}{\partial y}+\frac{\partial \theta}{\partial y} \frac{\partial A(H(x, y))}{\partial x}=1 .
$$

Therefore $A(H(x, y)), \theta(x, y)$ are the action-angle variables for the system (2.4). See [2, p. 279].

\section{The main theorem}

THEOREM 3.1. Given the area-preserving mapping (1.1)

$$
\begin{aligned}
& x_{1}=x+P(x, y), \\
& y_{1}=y+Q(x, y),
\end{aligned}
$$

where $P(x, y), Q(x, y)$ are real-analytic functions,

$$
P(x, y), Q(x, y)=O\left(r^{h}\right), \quad r^{2}=x^{2}+y^{2},
$$

$h>1$, near $(0,0)$ and $P^{2}+Q^{2} \neq 0$ in a small, punctured neighbourhood of $(0,0)$. Let $H$ be, as before,

$$
H(x, y) \stackrel{\text { def }}{\equiv} \int_{(0,0)}^{(x, y)}\left[Q-\frac{1}{2}\left(P Q_{x}-P_{x} Q\right)\right] d x-\left[P+\frac{1}{2}\left(P Q_{y}-P_{y} Q\right)\right] d y .
$$

The isolated parabolic fixed point $(0,0)$ is surrounded by closed invariant curves if the following conditions hold:

(a) $H(x, y)>0$ in a punctured neighborhood of the origin and the curves $H(x, y)=\lambda$, $\lambda>0$, are closed and surround the origin.

(b) There exist positive constants $c_{1}, c_{2}$ such that near the origin

$$
\begin{gathered}
x H_{x}+y H_{y} \geq c_{1} H, \\
0<x^{2} H_{x x}+2 x y H_{x y}+y^{2} H_{y y} \leq c_{2} H .
\end{gathered}
$$

(c) There exists an integer $k$,

$$
h<k<(4 h-2) \frac{h^{2}+2 h+1}{2 h^{2}+2 h+1}
$$

such that

$$
H(x, y) \geq c_{3} r^{k}>0
$$

near the origin.

The case $H<0$ is treated similarly.

Proof. Our aim is to show that under the change of variables $(x, y) \leftrightarrow(\rho, \theta)$, the mapping (1.1) actually becomes the twist mapping (2.1)

$$
\begin{aligned}
& \theta_{1}=\theta+\rho+f(\rho, \theta), \\
& \rho_{1}=\rho+g(\rho, \theta),
\end{aligned}
$$

where $f(\rho, \theta), g(\rho, \theta)$ are real-analytic, periodic in $\theta$ and $f=o(\rho), g=o\left(\rho^{2}\right)$ in a small neighbourhood of the origin. As several of our estimates depend on the area $A(\lambda)$ of the region $H \leq \lambda$, we begin with

LEMMA 1. If (3.1), (3.2) hold near the origin, then

$$
A^{\prime}(\lambda)=O(A(\lambda) / \lambda), \quad A^{\prime \prime}(\lambda)=O\left(A(\lambda) / \lambda^{2}\right) .
$$


Proof. Let us write the Hamiltonian in polar coordinates as $H(r, \phi)=\lambda$. Since $x y^{\prime}-y x^{\prime}=x H_{x}+y H_{y} \geq c_{1} H>0, H=\lambda$ is a starlike curve, and we can write $r=$ $r(\phi, \lambda)$ such that

$$
H(r(\phi, \lambda), \phi)=\lambda .
$$

Differentiating with respect to $\lambda$,

$$
\frac{\partial H}{\partial r} \frac{\partial r}{\partial \lambda}=1
$$

Now

$$
A(\lambda)=\frac{1}{2} \int_{H=\lambda} r^{2} d \phi=\frac{1}{2} \int_{0}^{2 \pi} r^{2}(\phi, \lambda) d \phi
$$

and since by (3.6), $r_{\lambda}=1 / H_{r}$

$$
A^{\prime}(\lambda)=\int_{0}^{2 \pi} r r_{\lambda} d \phi=\int_{0}^{2 \pi} r / H_{r} d \phi .
$$

But according to (3.1), $r H_{r}=x H_{x}+y H_{y} \geq c_{1} H$, therefore

$$
A^{\prime}(\lambda) \leq \int_{H=\lambda}\left(1 / c_{1} H\right) r^{2} d \phi=O(1 / \lambda) A(\lambda) .
$$

Similarly, by $r_{\lambda}=1 / H_{r}$

$$
\begin{aligned}
A^{\prime \prime}(\lambda) & =\int_{0}^{2 \pi}\left(r / H_{r}\right)_{r} r_{\lambda} d \phi=\int\left(H_{r}-r H_{r r}\right) / H_{r}^{3} d \phi \\
& =\int\left(\left(r H_{r}-r^{2} H_{r r}\right) /\left(r H_{r}\right)^{3}\right) r^{2} d \phi .
\end{aligned}
$$

According to (3.2), $r^{2} H_{r r}=x^{2} H_{x x}+2 x y H_{x y}+y^{2} H_{y y} \leq c_{2} H$, therefore

$$
A^{\prime \prime}(\lambda)=\int_{H=\lambda} O\left(H^{-2}\right) r^{2} d \phi=O\left(\lambda^{-2}\right) A(\lambda) .
$$

LEMMA 2. Let $H(x, y)>0$ in a punctured neighborhood of the origin. If the mapping (1.1) is written as in (2.5) and the homogeneous polynomial $x q(x, y)-y p(x, y)$ of degree $h+1$ has along any ray $y / x=$ constant a zero of order l at most, $0 \leq l \leq h+1$, then

$$
A(\lambda)= \begin{cases}O\left(\lambda^{2 /(h+1)}\right), & l<(h+1) / 2 \\ O\left(\lambda^{2 /(h+1)} \log \left(\lambda^{-1}\right)\right), & l=(h+1) / 2 \\ O\left(\lambda^{2 /(h+1)[1-1 /(h+1)+1 / 2 l]}\right), & l>(h+1) / 2 .\end{cases}
$$

Since the proof of this lemma is not closely related to the main idea of our theorem, it is delayed to the end of this section.

Let us return to the proof of the theorem. The point $(x, y)$ is mapped by (1.1) into $\left(x_{1}, y_{1}\right)=(x+P, y+Q)$. Let $H=\lambda$ and $H=\lambda+\Delta \lambda$ be curves through $(x, y)$ and $\left(x_{1}, y_{1}\right)$, respectively. The relative position of these curves depends, of course, on the point $(x, y)$, but as proved in Proposition 3, we always have $\Delta \lambda=O\left(r^{4 h-2}\right)$. By inequality (3.4), every point on the curve $H=\lambda$ satisfies $r=O\left(\lambda^{1 / k}\right)$ and so

$$
\Delta \lambda=O\left(\lambda^{(4 h-2) / k}\right) \text {. }
$$


But since $k<(4 h-2)\left(h^{2}+2 h+1\right) /\left(2 h^{2}+2 h+1\right)<4 h-2$, this implies

$$
\Delta \lambda=o(\lambda) \text {. }
$$

Consider now $T(\lambda)$. The worst bound of Lemma 2 for $A(\lambda)$ (which corresponds to $l=h+1)$ is

$$
A(\lambda)=O\left(\lambda^{(2 h+1) /(h+1)^{2}}\right) .
$$

Since $k$ is smaller than $(4 h-2)\left(h^{2}+2 h+1\right) /\left(2 h^{2}+2 h+1\right)$, it is easily seen that $(2 h+1) /(h+1)^{2}>2-(4 h-2) / k$. Consequently, $A(\lambda)=o\left(\lambda^{2-(4 h-2) / k}\right)$ and by Lemma $1, A^{\prime \prime}(\lambda)=o\left(\lambda^{-(4 h-2) / k}\right)$. So

$$
\begin{aligned}
T(\lambda+\Delta \lambda)-T(\lambda) & =T^{\prime}\left(\lambda_{0}\right) \Delta \lambda=A^{\prime \prime}\left(\lambda_{0}\right) O\left(\lambda^{(4 h-2) / k}\right) \\
& =o\left(\lambda_{0}^{-(4 h-2) / k}\right) O\left(\lambda^{(4 h-2) / k}\right)=o(1),
\end{aligned}
$$

since $\lambda<\lambda_{0}<\lambda+\Delta \lambda$ and $\lambda_{0}=\lambda(1+o(1))$. Note that this is by no means trivial, since $T(\lambda) \rightarrow \infty$ as $\lambda \rightarrow 0$. It rather shows how small $\Delta \lambda$ is. (The choice of the worst bound in Lemma 2 is not a severe restriction. If one takes the stricter bound $A(\lambda)=O\left(\lambda^{2 /(h+1)}\right)$, which corresponds to $l<(h+1) / 2$, it would enable us to extend the range of $k$ only by less than $1 / h$.)

Now we are ready to estimate the change of $\rho$ :

$$
\begin{aligned}
\rho_{1}-\rho & =1 / T\left(H\left(x_{1}, y_{1}\right)\right)-1 / T(H(x, y)) \\
& =-(T(\lambda+\Delta \lambda)-T(\lambda)) / T(\lambda) T(\lambda+\Delta \lambda) \\
& =o(1) / T^{2}(\lambda)=o\left(\rho^{2}\right) .
\end{aligned}
$$

Thus the second equation of (2.1) holds with $g(\rho, \theta)=o\left(\rho^{2}\right)$.

We turn to the first equation of $(2.1)$ and prove that

$$
\theta\left(x_{1}, y_{1}\right)-\theta(x, y)=\rho(1+o(1)) \text {. }
$$

Recall that the Hamiltonian system (2.4) generates a mapping $g^{t}:(x(0), y(0)) \rightarrow$ $(x(t), y(t))$. In particular after a time $t=1$, the point $(x, y)$ of $H=\lambda$ is mapped to the point $(x(1), y(1))$ of $H=\lambda$ :

$$
g^{1}:(x, y) \rightarrow(x(1), y(1)) .
$$

For these two points of $H=\lambda$, we have by (2.13)

$$
\theta(x(1), y(1))-\theta(x, y) \equiv \rho,
$$

thus instead of (3.9) it suffices to show that

$$
\theta(x(1), y(1))-\theta\left(x_{1}, y_{1}\right) \equiv o(\rho(x, y)),
$$

where, by (2.11)

$$
\begin{gathered}
\theta(x(1), y(1))=\rho(x(1), y(1)) \int_{H=\lambda}^{(x(1), y(1))} \frac{-y d x+x d y}{x H_{x}+y H_{y}}, \\
\theta\left(x_{1}, y_{1}\right)=\rho\left(x_{1}, y_{1}\right) \int_{H=\lambda+\Delta \lambda}^{\left(x_{1}, y_{1}\right)} \frac{-y d x+x d y}{x H_{x}+y H_{y}} .
\end{gathered}
$$


In (3.11) $\rho(x(1), y(1)) \equiv \rho(x, y)$ since both points lay on the same curve $H=\lambda$, while for $\rho\left(x_{1}, y_{1}\right)$ which appears in (3.12) we have already seen that $\rho\left(x_{1}, y_{1}\right)=$ $\rho(x, y)+o\left(\rho^{2}(x, y)\right)$. Dividing (3.10) by $\rho(x, y)$ results in

$$
\int_{H=\lambda}^{(x(1), y(1))} \frac{-y d x+x d y}{x H_{x}+y H_{y}}-(1+o(\rho)) \int_{H=\lambda+\Delta \lambda}^{\left(x_{1}, y_{1}\right)} \frac{-y d x+x d y}{x H_{x}+y H_{y}}=o(1) .
$$

Note that each integral in (3.13) equals a period of time in which a solution of (2.4) traverses an arc of a trajectory. As $T(\lambda) \rightarrow \infty$, each of these integrals is unbounded for $(x, y)$ near the origin. This is the source of the difficulty to verify (3.13).

We connect $\left(x_{1}, y_{1}\right),(x(1), y(1))$ by a straight line and the other two endpoints of the paths by a segment of the $x$-axis to get a closed path. On $y=0, x d y-y d x=0$, so (3.13) may be written as

$$
\int_{\left(x_{1}, y_{1}\right)}^{(x(1), y(1))} \frac{-y d x+x d y}{x H_{x}+y H_{y}}+\oint \frac{-y d x+x d y}{x H_{x}+y H_{y}}+o(\rho) \int_{H=\lambda+\Delta \lambda}^{\left(x_{1}, y_{1}\right)} \frac{-y d x+x d y}{x H_{x}+y H_{y}}=o(1),
$$

where the closed path consists, as explained above, from two arcs $H=\lambda, H=\lambda+\Delta \lambda$, an interval on the $x$-axis and another straight line.

We begin to estimate the third term of (3.14). By (3.1), $y x^{\prime}-x y^{\prime}=x H_{x}+y H_{y}>$ $c_{1} H>0$, hence $H=\lambda$ is starlike and $x d y-y d x$ has a fixed sign, say positive. Therefore, if we extend the integration to a whole period,

$$
\begin{aligned}
\left|o(\rho) \int_{H=\lambda+\Delta \lambda}^{\left(x_{1}, y_{1}\right)} \frac{-y d x+x d y}{x H_{x}+y H_{y}}\right| & \leq o(\rho) \oint_{H=\lambda+\Delta \lambda} \frac{-y d x+x d y}{x H_{x}+y H_{y}} \\
& =o(1 / T(\lambda)) T(\lambda+\Delta \lambda) \\
& =o((T(\lambda)+o(1)) / T(\lambda))=o(1) .
\end{aligned}
$$

Now we turn to the first term of (3.14). On a straight line $x d y-y d x$ has a fixed sign, say positive, so

$$
\left|\int_{\left(x_{1}, y_{1}\right)}^{(x(1), y(1))} \frac{-y d x+x d y}{x H_{x}+y H_{y}}\right| \leq O(1) \int_{\left(x_{1}, y_{1}\right)}^{(x(1), y(1))} \frac{-y d x+x d y}{H(x, y)} .
$$

The two endpoints lay on $H=\lambda, H=\lambda+\Delta \lambda$ respectively, but the whole segment does not lay necessarily between the two curves. However, by Proposition 2,

$$
\left[\left(x(1)-x_{1}\right)^{2}+\left(y(1)-y_{1}\right)^{2}\right]^{1 / 2}=O\left(r^{3 h-2}\right) .
$$

So, as in the proof of Proposition 3, the values of $H$ along the segment $(x(1), y(1))$, $\left(x_{1}, y_{1}\right)$ are

$$
\lambda+O\left(r^{h}\right) O\left(r^{3 h-2}\right)=\lambda+O\left(\lambda^{(4 h-2) / k}\right)=\lambda(1+o(1)) .
$$

(3.15) is, accordingly,

$$
O(1 / \lambda) \int_{\left(x_{1}, y_{1}\right)}^{(x(1), y(1))} x d y-y d x
$$

But the integral in (3.17) equals twice the area of the triangle with vertices $(0,0)$, $\left(x_{1}, y_{1}\right),(x(1), y(1))$, so it is not bigger than

$$
\begin{aligned}
{\left[x_{1}^{2}+y_{1}^{2}\right]^{1 / 2}\left[\left(x(1)-x_{1}\right)^{2}+\left(y(1)-y_{1}\right)^{2}\right]^{1 / 2} } & =r(1+o(1)) O\left(r^{3 h-2}\right)=O\left(r^{3 h-1}\right) \\
& =O\left(\lambda^{(3 h-1) / k}\right)
\end{aligned}
$$


Consequently, the quantity in (3.17) is $O\left(\lambda^{(-1+(3 h-1) / k}\right)=o(1)$, since

$$
k<(4 h-2)\left(h^{2}+2 h+1\right) /\left(2 h^{2}+2 h+1\right)<3 h-1 .
$$

Finally we estimate the middle term of (3.14). In this integral $x d y-y d x$ obviously changes its sign on the closed path, so we estimate its absolute value by transforming it to a multiple integral:

$$
\begin{aligned}
\oint \frac{-y d x+x d y}{x H_{x}+y H_{y}} & =\iint\left[\left(\frac{x}{x H_{x}+y H_{y}}\right)_{x}+\left(\frac{y}{x H_{x}+y H_{y}}\right)_{y}\right] d x d y \\
& =\iint\left[\frac{\left(x H_{x}+y H_{y}\right)-\left(x^{2} H_{x x}+2 x y H_{x y}+y^{2} H_{y y}\right)}{\left(x H_{x}+y H_{y}\right)^{2}}\right] d x d y .
\end{aligned}
$$

As we have seen in (3.16), the segment $(x(1), y(1)),\left(x_{1}, y_{1}\right)$ lies in a ring

$$
\lambda-O\left(\lambda^{(4 h-2) / k}\right)<H<\lambda+O\left(\lambda^{(4 h-2) / k}\right)
$$

and the whole domain of integration of (3.18) is included in such ring. By assumptions (3.1), (3.2), the integrand is $O(1 / \lambda)$, thus (3.18) is bounded by

$$
O(1 / \lambda)\left(A\left(\lambda+O\left(\lambda^{(4 h-2) / k}\right)\right)-A\left(\lambda-O\left(\lambda^{(4 h-2) / k}\right)\right)\right) \text {. }
$$

We have already seen that $A(\lambda)=o\left(\lambda^{2-(4 h-2) / k}\right)$ and by Lemma $1, A^{\prime}(\lambda)=$ $o\left(\lambda^{1-(4 h-2) / k}\right)$. Thus

$$
\begin{aligned}
A\left(\lambda+O\left(\lambda^{(4 h-2) / k}\right)\right)-A\left(\lambda-O\left(\lambda^{(4 h-2) / k}\right)\right) & =A^{\prime}\left(\lambda_{0}\right) O\left(\lambda^{(4 h-2) / k}\right) \\
& =o\left(\lambda_{0}^{1-(4 h-2) / k}\right) O\left(\lambda^{(4 h-2) / k}\right)=o(\lambda)
\end{aligned}
$$

since $\lambda_{0}=\lambda(1+o(1))$. To summarize, the integral in (3.18) is $o(1)$, too. This completes to establish the twist mapping (2.1)

$$
\begin{aligned}
& \theta_{1}=\theta+\rho+f(\rho, \theta), \\
& \rho_{1}=\rho+g(\rho, \theta),
\end{aligned}
$$

with $f(\rho, \theta)=o(\rho), g(\rho, \theta)=o\left(\rho^{2}\right)$ near $\rho=0 . f(\rho, \theta)$ and $g(\rho, \theta)$ are obviously analytic when $\rho>0$ and have period 1 .

A small neighborhood $0<H(x, y)<\psi^{-1}(\varepsilon)$ of the origin is thus mapped on a narrow annulus $0<\rho<\varepsilon$. When we replace $\rho$ by $\varepsilon \rho$, we get

$$
\begin{aligned}
& \theta_{1}=\theta+\varepsilon \rho+\tilde{f}, \\
& \rho_{1}=\rho+\tilde{g},
\end{aligned}
$$

on the ring $0<\rho<1$. Here $\tilde{f}(\rho, \theta)=f(\varepsilon \rho, \theta)=o(\varepsilon), \tilde{g}(\rho, \theta)=\varepsilon^{-1} g(\varepsilon \rho, \theta)=o(\varepsilon)$. Hence $(|\tilde{f}|+|\tilde{\mathbf{g}}|) / \varepsilon \rightarrow 0$ as $\varepsilon \rightarrow 0$, as required in the theorem of Moser. Finally, since mapping (1.1) is area preserving, each closed curve which surrounds the fixed point $(0,0)$, intersects its image by (1.1). Thus, all the details of Moser's theory apply to our twist mapping and the existence of closed invariant curves is proved.

Among our assumptions, (3.3) seems to be the least natural. It would be interesting to obtain another proof in which this condition could be relaxed or dispensed with.

Proof of Lemma 2. Recall that by (2.6),

$$
H(x, y)=(x q-y p) /(h+1)+O\left(r^{h+2}\right) \text {. }
$$


The assumption $H>0$ implies that $x q-y p \geq 0$ near the origin. Let us write the equation of the curve $H=\lambda$ as

$$
r^{h+1} R(\phi)+O\left(r^{h+2}\right)=\lambda,
$$

where $R(\phi)=(\cos \phi q(\cos \phi, \sin \phi)-\sin \phi p(\cos \phi, \sin \phi)) /(h+1) \geq 0 . R(\phi)$ may have a finite number of zeros, one for each ray on which the homogeneous polynomial $x q-y p$ vanishes. For simplicity let us assume that $R(\phi)$ has a single zero located at $\phi=0$ and its multiplicity is $l, 0 \leq l \leq h+1$. The general case is treated similarly.

Since $H \leq c_{1} r^{h+1}$, on $H=\lambda$ one has $r \geq c_{2} \lambda^{1 /(h+1)}$ and by (3.19)

$$
\begin{gathered}
r^{2}=\lambda^{2 /(h+1)}(R(\phi)+O(r))^{-2 /(h+1)} \geq \lambda^{2 /(h+1)}\left(R(\phi)+c_{3} \lambda^{1 /(h+1)}\right)^{-2 /(h+1)}, \\
A(\lambda)=\frac{1}{2} \int_{-\pi}^{\pi} r^{2} d \phi \leq\left(\frac{1}{2}\right) \lambda^{2 /(h+1)} \int_{-\pi}^{\pi}\left(R(\phi)+c_{3} \lambda^{1 /(h+1)}\right)^{-2 /(h+1)} d \phi .
\end{gathered}
$$

By our assumptions, $R(\phi)=\phi^{l} K(\phi), c_{4} \leq K(\phi) \leq c_{5}$, on $[-\pi, \pi]$. Hence

$$
A(\lambda)=O\left(\lambda^{2 /(h+1)}\right) \int_{-\pi}^{\pi}\left(\phi^{l}+c_{6} \lambda^{1 /(h+1)}\right)^{-2 /(h+1)} d \phi .
$$

If $l<(h+1) / 2$, the integral in (3.20) is obviously smaller than $\int_{-\pi}^{\pi} \phi^{-2 l /(h+1)} d \phi=$ $O(1)$. If $l>(h+1) / 2$, we divide $[0, \pi]$ into $\left[0, \lambda^{1 / l(h+1)}\right] \cup\left[\lambda^{1 / /(h+1)}, \pi\right]$. The corresponding integral of $(3.20)$ is

$$
\int_{0}^{\lambda^{1 / /(h+1)}}\left(\phi^{l}+c_{6} \lambda^{1 /(h+1)}\right)^{-2 /(h+1)} d \phi+\int_{\lambda^{1 /(h+1)}}^{\pi}\left(\phi^{l}+c_{6} \lambda^{1 /(h+1)}\right)^{-2 /(h+1)} d \phi
$$

plus similar terms for $[-\pi, 0]$. The first of these integral is obviously $O\left(\lambda^{1 / l(h+1)} \lambda^{-2 /(h+1)^{2}}\right)$ and the second one is smaller than

$$
\int_{\lambda^{1 /(h+1)}}^{\pi} \phi^{-2 l /(h+1)} d \phi=O\left(\lambda^{[1-2 l /(h+1)] / /(h+1)}\right)=O\left(\lambda^{1 / l(h+1)} \lambda^{-2 /(h+1)^{2}}\right)
$$

and the estimate $A(\lambda)=O\left(\lambda^{2 /(h+1)[1-1 /(h+1)+1 / 2 l]}\right)$ follows.

When $l=(h+1) / 2$, the result is proved by the same method.

\section{Examples}

The following is the most simple particular case of Theorem 3.1.

THEOREM 4.1. Suppose that the real analytic, area-preserving mapping (1.1) can be written in a neighborhood of $(0,0)$ as

$$
\begin{aligned}
& x_{1}=x+p(x, y)+\hat{p}(x, y), \\
& y_{1}=y+q(x, y)+\hat{q}(x, y),
\end{aligned}
$$

where $p(x, y), q(x, y)$ are polynomials homogeneous of degree $h, h>1$, and $\hat{p}, \hat{q}$ are such that

$$
\hat{p}(x, y), \hat{q}(x, y)=O\left(r^{h+1}\right) \quad \text { near } r=0 .
$$

If

$$
x q(x, y)-y p(x, y) \neq 0 \text { for }(x, y) \neq(0,0)
$$

then the fixed point $(0,0)$ is surrounded by closed invariant curves. 
Proof. We have already seen that if the mapping (1.1) is written near $(0,0)$ as $(4.1)$, then, (2.6)

$$
H(x, y)=(x q-y p) /(h+1)+O\left(r^{h+2}\right) .
$$

If in addition we assume now that the $(h+1)$ th order homogeneous polynomial $x q(x, y)-y p(x, y)$ does not vanish for $(x, y) \neq(0,0)$, then

$$
|x q-y p| \geq A r^{h+1}>0
$$

and consequently

$$
H(x, y)=\frac{x q-y p}{h+1}(1+o(1)) .
$$

Now all the conditions of Theorem 3.1 are satisfied with $k=h+1$. Indeed,

$$
\begin{gathered}
H(x, y) \geq c r^{h+1}, \\
x H_{x}+y H_{y}=(x q-y p)+O\left(r^{h+2}\right)=(h+1) H(x, y)(1+o(1)), \\
x^{2} H_{x x}+2 x y H_{x y}+y^{2} H_{y y}=h(x q-y p)+O\left(r^{h+2}\right)=h(h+1) H(x, y)(1+o(1))
\end{gathered}
$$

and (3.3), (3.4) hold trivially with $k=h+1$.

The same result may be deduced by the theorem of Simo [5]. If we want to write (4.1) as $x_{1}=\partial g / \partial y_{1}, y=\partial g / \partial x$, we get

$$
\begin{gathered}
x_{1}=x+p(x, y)+O\left(r^{h+1}\right)=x+p\left(x, y_{1}\right)+O\left(r^{h+1}\right)=\partial g / \partial y_{1}, \\
y=y_{1}-q(x, y)+O\left(r^{h+1}\right)=y_{1}-q\left(x, y_{1}\right)+O\left(r^{h+1}\right)=\partial g / \partial x .
\end{gathered}
$$

By the argument that was used to show (2.6), it follows now that

$$
g\left(x, y_{1}\right)=x y_{1}-\frac{x q-y_{1} p}{h+1}+O\left(r^{h+1}\right)
$$

and the result follows.

Some variations on the theme of Theorem 4.1 will appear elsewhere.

Example 1. (Continued.) For the area-preserving mapping

$$
\begin{aligned}
& x_{1}=x+y^{2 n-1}, \\
& y_{1}=y-\left(x+y^{2 n-1}\right)^{2 n-1},
\end{aligned}
$$

we have $x q-y p=x^{2 n}+y^{2 n}$. Hence, by Theorem 4.1, it really has closed invariant curves, as hinted by figure 1 .

Example 2. (Continued.) That the mapping

$$
\begin{aligned}
& x_{1}=x-2 x^{2} y+4 x y^{3}+x^{4}-2 y^{5}-4 x^{3} y^{2}+\cdots+y^{8}, \\
& y_{1}=y+2 x y^{2}-4 x^{3} y-y^{4}+2 x^{5}+8 x^{2} y^{3}+\cdots+y^{16},
\end{aligned}
$$

does not satisfy the conditions of Theorem 4.1 since $x q-y p=4 x^{2} y^{2}$ is not positive definite. However, it does satisfy the conditions of Theorem 3.1 with $h=3, k=6$. Here

$$
\begin{gathered}
H(x, y)=x^{2} y^{2}-\left(x y^{4}+x^{4} y\right)+\frac{1}{3}\left(x^{6}+y^{6}\right)+2 x^{3} y^{3}+O\left(r^{7}\right), \\
x H_{x}+y H_{y}=4 x^{2} y^{2}-5\left(x y^{4}+x^{4} y\right)+2\left(x^{6}+y^{6}\right)+12 x^{3} y^{3}+O\left(r^{7}\right), \\
x^{2} H_{x x}+2 x y H_{x y}+y^{2} H_{y y}=12 x^{2} y^{2}-20\left(x y^{4}+x^{4} y\right)+10\left(x^{6}+y^{6}\right)+60 x^{3} y^{3}+O\left(r^{7}\right) .
\end{gathered}
$$


We can write

$$
\begin{aligned}
& A x^{2} y^{2}-B\left(x y^{4}+x^{4} y\right)+C\left(x^{6}+y^{6}\right)+D x^{3} y^{3} \\
& =\alpha x^{2} y^{2}[1+\varepsilon x y]+\left[\beta x y-\gamma\left(x^{3}+y^{3}\right)\right]^{2}+\delta\left[x^{6}+y^{6}\right],
\end{aligned}
$$

with $A=\alpha+\beta^{2}, B=2 \beta \gamma, C=\gamma^{2}+\delta$. It is easily seen that $\alpha, \delta$ are positive if $A, C>0$ and $B^{2} / 4 C<\beta^{2}<A$, that is $B^{2}<4 A C$. $H(x, y)$ satisfies these conditions, so

$$
H(x, y)>(\delta / 2)\left(x^{6}+y^{6}\right)>c r^{6}
$$

near the origin. Similar arguments show that

$$
\begin{gathered}
x H_{x}+y H_{y} \geq 2 H, \\
0<x^{2} H_{x x}+2 x y H_{x y}+y^{2} H_{y y} \leq 50 H .
\end{gathered}
$$

Finally, (3.3), (3.4) hold with $h=3, k=6$.

Acknowledgement. This research was supported by the Fund for the Promotion of Research at the Technion.

\section{REFERENCES}

[1] V. I. Arnold. Mathematical Methods of Classical Mechanics. Springer-Verlag, Berlin, 1978.

[2] J. Moser. On invariant curves of area-preserving mappings of an annulus. Nachr. Math. Wiss., Gottingen II, Math. Phys. K1 (1962), 1-20.

[3] C. L. Siegel \& J. Moser. Lectures on Celestial Mechanics. Springer Verlag, Berlin, 1971.

[4] C. Simo. Stability of degenerate fixed points of analytic area preserving mappings. Astérisque 98-99 (1982), 184-194.

[5] C. Simo. Invariant curves near parabolic points and regions of stability. Lecture Notes in Mathematics 819 pp. 418-424, Springer Verlag, Berlin, 1980. 